\title{
Small Perturbations in General Relativity: Tensor Harmonics of Arbitrary Symmetry
}

\author{
R.A. Konoplya \\ Department of Physics, Dnepropetrovsk State University, \\ per. Nauchny 13, Dnepropetrovsk, 49050 Ukraine
}

March 12, 2018

\begin{abstract}
We develop a method for constructing of the basic functions with witch to expand small perturbations of space-time in General Relativity. The method allows to obtain the tensor harmonics for perturbations of the background space-time admitting an arbitrary group of isometry, and to split the linearized Einstein equations into the irreducible combinations. The essential point of the work is the construction of the generalized Casimir operator for the underlying group, which is defined not only on vector but also on tensor fields. It is used to construct the basic functions for spaces of tensor representations of the background metric's group of isometry. The method, being general, is applied here to construction of the basic functions for the case of the threeparameter group of isometry $G_{3}$ acting on the two-dimensional non-isotropic surface of transitivity. As quick illustrations of the method we consider the well-known particular cases: cylindrical harmonic for the flat spacetime, and Regge-Wheller spherical harmonics for the Schwarzschild metric.
\end{abstract}

\section{Introduction}

There are the two widely accepted ways to construct tensor harmonics. The first is based on the construction of the tensor basis, corresponding to a given underlying symmetry of space [1]. The second way is to consider the action of the complete set of the invariant commutative operators on scalar harmonics of space. However each of these methods, being well developed in General Relativity for spaces of high symmetries, such as spherical [2], 3] for example, encounters serious difficulties when dealing with spaces of low symmetry. In the last few years the perturbation formalism in General Relativity has attracted some attention for a possibility of detecting gravitational waves from astrophysical sources by antennas. This stimulated the development of the second order perturbation formalism for Schwarzschild black holes and, in this connection, a review of the first one (see [4] and references therein).

In the present work we propose the method of the obtaining tensor harmonics that enormously simplifies calculations for first order perturbations in General Relativity and promises some advantages when dealing with the second order. In [5], it was proposed the natural generalization of the Casimir operator defined so that the Lie derivatives are put instead of the corresponding infinitesimal operators (see formula (11) of the present work). The metric used in the definition admits a group of isometry stipulated by the invariance condition for this operator. The generalized Casimir operator is invariant in the space of tensor functions. This made it possible to formulate correctly and solve the eigenvalue and tensor eigenfunction problems, and, thereby, to construct tensor representations of various groups. However, if we follow this approach "directly", the system of differential equations generated by a given generalized Casimir operator appears with "tangled" components of tensor functions, and thus the direct solving of the tensor eigenfunction problem, in general case becomes impossible. An accurate and natural way to "disentangle" such system of differential equations (i.e. to separate variables) is to use invariant technique developed when considering decomposition of the tangent bundle of pseudo-Riemannian manifolds into the corresponding subbundles [6].

When at last the set of basic functions for a given symmetry of the background metric are obtained we can expand the small perturbations of a metric in terms of these basic functions, substitute such an expanded metric for that into the linearized Einstein equations, and try to separate variables into them. For an Abelian underlying group of symmetry owing to the Lie theorem it becomes obvious that the splitting of the linearized Einstein equations into irreducible combinations eventually leads to separations of the corresponding variables. However in the general case we can not know beforehand whether variables will be separated or not. 
The paper is organized as follows. In Sec.II we give, briefly, the method for constructing of tensor harmonics and for obtaining of the linearized Einstein equations in form of irreducible combinations. In Sec.III the tensor harmonics are obtained for the case of three-parameter group on the two-dimensional non-isotropic surface of transitivity. Sec.IV deals with the two particular cases of the previous section: cylindrical waves as perturbations of the flat space-time and spherical Regge-Wheeler waves.

\section{The method}

Let $M^{n}$ be an $n$-dimensional manifold and $G^{r}$ is an $r$-parameter transformation group on $M^{n}$. Note, that unlike the Lie algebra of the tangent to one-parameter subgroups of the group $G^{r}$ vectors $\xi_{i}$, defined only on functions, that of the Lie derivatives defined on tensors of an arbitrary type, are invariant operators $L_{\xi_{i}}$ under the general coordinate transformations. Hence it seems natural to define the generalized Casimir operator of the second order as

$$
G=\gamma^{i k} L_{\xi_{i}} L_{\xi_{k}},
$$

where $\gamma^{i k}(i, k=1,2, \ldots r)$ are contravariant components of some unknown metric, which is subject to be determined. Since this operator commutes with all the operators $L_{\xi_{i}}$ of the representation we have

$$
\left(L_{\xi_{j}} \gamma^{-1}\right)^{i k} \equiv \xi_{j} \gamma^{i k}+C_{j l}^{i} \gamma^{l k}+C_{j l}^{k} \gamma^{i l}=0,
$$

where the tensor

$$
\gamma^{-1}=\gamma^{i k} \xi_{i} \otimes \xi_{k} \quad\left(\operatorname{rank}\left\|g^{-1}\right\|=s\right)
$$

defines the metric on covectors belonging to the surfaces of transitivity $M^{s} \subset M^{n}(s \leq r, s \leq n)$. Owing to (2), the group $G^{r}$ on the surfaces of transitivity is a group of isometries, where $\vec{\xi}_{i}$ are the Killing's vectors. Solutions of the Killing's equations (2) give us the metric for the definition of the generalized Casimir operator (11). It turns out that for semisimple groups that will be enough to consider only constant solutions of (2). In the general case the consideration of the constant solutions of (2) will be inadequate for construction of the generalized Casimir operator $G$, which is non-degenerate on $M^{s}$.

A tensor $T$ of type $(p, q)$ on $M^{s}$ will be the eigenfunction tensor of the generalized Casimir operator $G$, provided the equation

$$
G T \equiv \gamma^{i k} L_{\xi_{i}} L_{\xi_{k}} T=\lambda T .
$$

is satisfied. This equation can be rewritten in the coordinate form

$$
\mathcal{G} T_{b_{1} \ldots b_{q}}^{a_{1} \ldots a_{p}} \equiv \gamma^{i k} \mathcal{L}_{\xi_{i}} \mathcal{L}_{\xi_{k}} T_{b_{1} \ldots b_{q}}^{a_{1} \ldots a_{p}}=\lambda T_{b_{1} \ldots b_{q}}^{a_{1} \ldots a_{p}},
$$

where $\mathcal{G} T_{b_{1} \ldots b_{q}}^{a_{1} \ldots a_{p}} \equiv(G T)_{b_{1} \ldots b_{q}}^{a_{1} \ldots a_{p}}$ are representations of the generalized Casimir operator acting on the tensor $T$ in an arbitrary vector basis $e_{a}\left(e_{a}(a=1,2, \ldots, s)\right.$ and $e^{a}, e^{a}\left(e_{b}\right)=\delta_{b}^{a}$, are vector and co-vector bases on $M^{s}$ respectively), $T_{b_{1} \ldots b_{q}}^{a_{1} \ldots a_{p}}$ are the components of the tensor $T$ with respect to the basis $e_{a}$.

Since the Lie operators "tangle" components of $T$, it is difficult to solve the equation (5) directly. In order to solve the system of equations (5) it is necessary to "disentangle" components of the tensor $T$ in the equations, i.e. to diagonalize the operator $G$. The dioganalization of the generalized Casimir operator $G$ can be realized invariantly. Herewith the tensor equations (5D) split into the system of scalar differential equations for irreducible components of the tensor $T$.

Following [6], we shall say that a split structure $\mathcal{H}^{s}$ is introduced on $M$ if the $s$ linear symmetric operators (projectors) $H^{a}(a=1,2, \ldots s)$ of a constant rank with the properties

$$
H^{a} \cdot H^{b}=\delta^{a b} H^{b} ; \quad \sum_{a=1}^{s} H^{a}=I,
$$

where $I$ is the unit operator $(I \cdot X=I, \quad \forall X \in T(M))$, are defined on $T(M)$. Here we consider a linear operator $L$ on the tangent bundle $T(M)$ as a tensor of type $(1,1)$ for which $L \cdot X \equiv L(X) \in T(M), \forall X \in T(M)$. The product of two linear operators $L \cdot H$ obeys the rule $(L \cdot H) \cdot X=L \cdot(H \cdot X) \in T(M), \forall X \in T(M)$. An operator $H$ is called a symmetric one if $(H \cdot X, Y)=(X, H \cdot Y), \forall X, Y \in T(M)$.

Then we can obtain the decomposition the tangent bundle $T(M)$ and cotangent bundle $T^{*}(M)$ into the $\left(n_{1}+\right.$ $\left.n_{2}+\ldots+n_{s}\right)$ subbundles $\Sigma^{a}, \Sigma_{a}^{*}$, so that

$$
T(M)=\bigoplus_{a=1}^{s} \Sigma^{a} ; \quad T^{*}(M)=\bigoplus_{a=1}^{s} \Sigma_{a}^{*}
$$


Arbitrary vectors, covectors, and metrics are decomposed according to the scheme:

$$
X=\sum_{a=1}^{s} X^{a}, \quad \omega=\sum_{a=1}^{s} \omega_{a}, \quad g=\sum_{a=1}^{s} g^{a}, \quad g^{-1}=\sum_{a=1}^{s} g_{a}^{-1}
$$

where $X^{a}=H^{a} \cdot X, \quad H^{b} \cdot X^{a}=0, \quad X^{a} \cdot X^{b}=0, \quad \omega_{a}=\omega \cdot H^{a}, \quad \omega_{a}\left(X^{b}\right)=0, \quad(a \neq b)$.

Using this scheme we can obtain the decomposition of more complex tensors.

We shall say that a split structure $\mathcal{H}^{s}$ is compatible with a group of isometries if the conditions of invariance of $\mathcal{H}^{s}$ are satisfied, i.e. if

$$
L_{\xi_{i}} H^{a}=0, \quad(i=1,2, \ldots r ; a=1,2, \ldots s) .
$$

The equations (6), (9) define the invariant projection tensors.

In order to construct the projectors we require existence of such a dual vector $e_{a}$ and co-vector $e^{b}$ bases on $M^{s}$, that

$$
e_{a} \cdot e^{b}=\delta_{a}^{b} ; \quad H^{a}=e_{a} \otimes e^{a} .
$$

From now and on there is no summation on repeating indices $a$ and $b$. The invariance condition of (9) yields

$$
\left(L_{\xi_{i}} e^{a}\right) \cdot e_{b}=0 \quad(a \neq b)
$$

Hence it follows

$$
L_{\xi_{i}} e^{a}=\mu_{i}^{a} e^{a}, \quad L_{\xi_{i}} e_{a}=-\mu_{i}^{a} e_{a},
$$

where the factors of proportionality $\mu_{i}^{a}$ are some functions, satisfying the equation

$$
\xi_{i} \mu_{k}^{a}-\xi_{k} \mu_{i}^{a}=C_{i k}^{j} \mu_{j}^{a}
$$

which follows from the integrability condition of (12). Thus, the problem of the construction of the invariant projectors reduces to construction of the dual vector $\left\{e_{a}\right\}$ and covector $\left\{e^{b}\right\}$ bases satisfying the corresponding systems of equations in (12). Some of the factors $\mu_{i}^{a}$, or even all of them in some cases, can vanish. Then the projectors are constructed by means of the invariant basis $\left\{e_{a}: L_{\xi_{i}} e_{a}=0\right\}$. In particular, for simply transitive groups $(r=s)$, the invariant vector basis $\left\{e_{a}\right\}$ can be expressed in the form

$$
e_{a}=L_{a}^{b} \xi_{b} \quad\left(\operatorname{det}\left\|L_{a}^{b}\right\| \neq 0\right) .
$$

where the factors $L_{a}^{b}$ satisfy the equations $\xi_{b} L_{d}^{a}+C_{b q}^{a} L_{d}^{q}=0$. The integrability conditions of these equations are satisfied owing to the Jacobi identity. Using the last equations it can easily be shown that the tensor $g^{-1}$, constructed by the formula

$$
g^{-1}=\delta^{a b} e_{a} \otimes e_{b}=g^{a b} \xi_{a} \otimes \xi_{b} ; \quad g^{a b}=L_{c}^{a} L_{d}^{b} \delta^{c d} .
$$

actually satisfies the Killing's equations (2), and thereby will be the inverse metric (3).

Now we shall return to the general case of arbitrary bases $\left\{e_{a}, e^{b}\right\}$. The initial tensor $T$ can be expanded in the series

$$
T=\sum_{A, B} \hat{T}_{B}^{A}=\sum_{A, B} T_{B}^{A} \hat{e}_{A}^{B}
$$

where $\left\{\hat{e}_{A}^{B}\right\}=\left\{e_{a_{1}} \otimes \cdots \otimes e_{a_{p}} \otimes e^{b_{1}} \otimes \cdots \otimes e^{b_{q}}\right\}$ is the tensor basis, $\hat{T}_{B}^{A}=T_{B}^{A} e_{A}^{B}$ is the tensor monomial and $T_{B}^{A} \equiv T_{b_{1} \ldots b_{q}}^{a_{1} \ldots a_{p}}$ is its component. $A=\left\{a_{1}, \ldots, a_{p}\right\}$ and $B=\left\{b_{1}, \ldots, b_{q}\right\}$ are collective indices. The sum in (16) comprises the complete set of indices $A, B$. It is easy to show that since the projectors $H_{a}$ are invariant, the eigenvalue equations (4) and (5) split into the set of independent eigenvalue invariant equations for monomials

$$
G \hat{T}_{B}^{A} \equiv \gamma^{i k} L_{\xi_{i}} L_{\xi_{k}} \hat{T}_{B}^{A}=\lambda \hat{T}_{B}^{A} .
$$

Using this relation together with (12) and (13) we obtain

$$
\mathcal{G} T_{B}^{A} \equiv \gamma^{i k} \mathcal{L}_{\xi_{i}} \mathcal{L}_{\xi_{k}} T_{B}^{A}=\gamma^{i k}\left(\xi_{i}-\phi_{i B}^{A}\right)\left(\xi_{k}-\phi_{k B}^{A}\right) T_{B}^{A}=\lambda T_{B}^{A} .
$$

Here

$$
\phi_{i B}^{A}=\sum_{k=1}^{p} \mu_{i}^{a_{k}}-\sum_{n=1}^{q} \mu_{i}^{b_{n}}, \quad A=\left\{a_{1}, \ldots, a_{p}\right\}, \quad B=\left\{b_{1}, \ldots, b_{q}\right\}
$$


In order that the tensor equation (44) could go over into the invariantly split equations (17) for the irreducible components $T_{B}^{A}$, we must make a change $T \rightarrow T_{B}^{A} ; L_{\xi_{i}} \rightarrow \mathcal{L}_{\xi_{i}}=\xi_{i}-\phi_{i B}^{A}$. The equations (17) can be rewritten in the form

$$
\mathcal{G} T_{B}^{A}=\left[K-2 \gamma^{i k} \phi_{i B}^{A} \xi_{k}-\gamma^{i k} \xi_{i} \phi_{k B}^{A}+\gamma^{i k} \phi_{i B}^{A} \phi_{k B}^{A}\right] T_{B}^{A}=\lambda T_{B}^{A},
$$

where $K=\gamma^{i k} \xi_{i} \xi_{k}$ is the standard Casimir operator defined in the space of scalar functions. The solutions of the equations (19) and (12), (13) give us the basic tensor functions $\hat{T_{B}^{A}}=T_{B}^{A} \hat{e}_{A}^{B}$ in the space of a tensor representation of the group $G^{r}$ (or, in other words, tensor harmonics). Note that if there exists the invariant basis (14), then the generalized Casimir operator (4) with respect to this basis reduces to the standard Casimir operator $K$, and in order to construct the tensor basis of representation that will be enough to determine the basis of representation in the space of scalar functions.

We proceed to apply this method to small perturbations in General Relativity. Let $g^{\mu \nu}$ be the background metric admitting some group of isometry generated by the set of arbitrary Killing vectors $\left.\xi_{i}\left(\mathcal{L}_{\xi\rangle}\right\}^{\mu \nu}=\prime\right)$, and $h_{\mu \nu}$ the perturbation. Small perturbations satisfy the linearized Einstein equations $\delta R_{\mu \nu}=-8 \pi G \delta S_{\mu \nu}$, where $S_{\mu \nu}=T_{\mu \nu}-(1 / 2) g^{\mu \nu} T_{\lambda}^{\lambda}$. The perturbation of the Ricci tensor $\delta R_{\mu \nu}$ satisfies the tensor relation [7]

$$
\delta R_{\mu \nu}=\frac{1}{2} g^{\lambda \rho}\left[h_{\lambda \rho ; \mu ; \nu}-h_{\rho \mu ; \nu ; \lambda}-h_{\rho \nu ; \mu ; \lambda}+h_{\mu \nu ; \rho ; \lambda}\right] .
$$

The perturbation of the metric and Ricci tensor, being tensors, can be written with respect to the basis constructed in accordance with (11)-(12) and extended to the full manifold $M^{4}$

$$
\begin{gathered}
h=h_{i k} e^{i} \otimes e^{k}+h_{j s}\left(e^{r} \otimes e^{s}+e^{s} \otimes e^{j}\right)+h_{s s^{\prime}} e^{s} \otimes e^{s^{\prime}}, \\
\delta R=\delta R_{i k} e^{i} \otimes e^{k}+\delta R_{j s}\left(e^{r} \otimes e^{s}+e^{s} \otimes e^{j}\right)+\delta R_{s s^{\prime}} e^{s} \otimes e^{s^{\prime}} .
\end{gathered}
$$

where $\mathrm{i}, \mathrm{k}, \mathrm{j}, \ldots$ are the transversal indices, s,s' denote vectors on the surface of transitivity on which the underlying group of isometry acts. The basis $\left(e^{i}, e^{s}\right)=e^{a},(a=i, s=0,1,2,3)$ we shall call a representation basis. The relations (21)-(22) represent the expansion of these tensors in the terms of the irreducible components $\delta R_{i k}$, $\delta R_{j s}, \delta R_{s s^{\prime}}, h_{i k}, h_{j s}, h_{s s^{\prime}}$. These components for the perturbation of the metric can be found by using the above procedure. To find them for the perturbation of the Ricci tensor we need to write the tensor relation (20) with respect to the representation basis. For this purpose one should substitute the components $h_{\mu \nu}$ for their "irreducible representatives" $h_{j k}, h_{j s}, h_{s s^{\prime}}$, and use the Christoffel symbols $\Gamma_{a b}^{c}:\left(\nabla_{e_{a}} e_{b}=\Gamma_{a b}^{c} e_{c}\right)$ with respect to the canonical basis when dealing with the covariant derivatives in (20) (for the formula (22) written out in terms of the basis vectors and of the Christoffel symbols see Appendix A). When considering space-times with matter one should express the source term $S_{\mu \nu}$ in the irreducible form as well.

In oder to simplify the form of the perturbations $h_{a b}$ one can use the gauge freedom, expanding the gauge vector in terms of the obtained basic functions belonging to the same eigenvalue as the corresponding perturbations of the metric: $\xi=\xi^{c} e_{c}$, where $\xi^{c}$, being proportional to the basic functions, are adjusted to simplify $h_{a b}$. Then the gauge transformations read

$$
h_{a b}^{\prime}=h_{a b}+\mathcal{L}_{\xi^{c} e_{c}} h_{a b}
$$

\section{Tensor Harmonics For $G_{3}$ Groups on $M^{2}$ surfaces of transitivity with non-isotropic Killing vectors}

The method, being general, can easily be applied to construction of tensor harmonics for fields of gravity admitting an arbitrary group of isometry. We shall consider here one of the most physically interesting cases, including flat and spherical symmetries, when a three-parameter group acts on a two dimensional surface of transitivity.

As is known the orbits $M^{2}$ of the group of isometry $G_{3}$ are the spaces of the constant Gauss curvature $\mathbf{K}$, (and the space-time $M^{4}$ is of type either $D$ or 0 by Petrov [8]). Fields of gravity admitting the $G_{3}$ group of isometry acting on the two-dimensional non-isotropic surfaces of transitivity can be divided into the six canonical types : $G_{3} V I, G_{3} V I I\left(\mathbf{K}=0\right.$; the signatures on $M^{2}$ are +- and ++ respectively), and $G_{3} V I I I$ and $G_{3} I X$ (four types) [9] [8]. From now and on we shall denote an arbitrary set of coordinates as $x, y, z$.

(a) $G_{3} V I$ and $G_{3} V I I$ on $M^{2}$. The Killing vectors have the form: $\xi_{1}=\partial_{x}, \xi_{2}=\partial_{y}, \xi_{3}=x \partial_{y}+\varepsilon y \partial_{x}$, where $\varepsilon= \pm 1$ for the groups $G_{3} V I$ and $G_{3} V I I$ respectively. They can be expressed in a more appropriate form in the cylindrical coordinates on Euclidean (for $\left.G_{3} V I I\right)$ and pseudo-Euclidean $\left(G_{3} V I\right)$ planes which we shall denote in both cases as $r, \varphi$.

$$
\xi_{1}=-\cos \varphi \partial_{r}+r^{-1} \sin \varphi \partial_{\varphi}, \quad \xi_{2}=-\sin \varphi \partial_{r}-r^{-1} \cos \varphi \partial_{\varphi}, \quad \xi_{3}=-\partial_{\varphi}
$$




$$
\xi_{1}=-\cosh \varphi \partial_{r}+r^{-1} \sinh \varphi \partial_{\varphi}, \quad \xi_{2}=\sinh \varphi \partial_{r}-r^{-1} \cosh \varphi \partial_{\varphi}, \quad \xi_{3}=-\partial_{\varphi}
$$

These groups are integrable and not semi-simple; the corresponding Cartan tensors are degenerate. The solution of the Killing equations in the cylindrical coordinates gives the metric for construction of the Casimir operator: $\gamma^{11}=-\gamma^{22}=1$, for $G_{3} V I$ and $\gamma^{11}=\gamma^{22}=1, \gamma^{33}=0$ for $G_{3} V I I$. The basis

$$
e_{1}=e^{c \varphi}\left(\partial_{r}+c r^{-1} \partial_{\varphi}\right), \quad e_{2}=e^{-c \varphi}\left(\partial_{r}-c r^{-1} \partial_{\varphi}\right)
$$

where $c=1$ for $G_{3} V I$ and $c=i$ for $G_{3} V I I$, can be chosen as the representation one on the surface of transitivity, for it satisfies (11)-(12) at $\mu_{3}^{1}=-c, \mu_{3}^{2}=c$. In this case the construction of tensor harmonics reduces to that of scalar harmonics. The latter are the eigenfunctions of the operators:

$$
\xi_{3} t_{\mu}^{\lambda}=-\partial_{\varphi} t_{\mu}^{\lambda}=-i \mu t_{\mu}^{\lambda}
$$

and

$$
\begin{gathered}
K t_{\mu}^{\lambda}=\left(\xi_{1}^{2}-\xi_{2}^{2}\right) t_{\mu}^{\lambda}=-\left(\partial_{r}^{2}+r^{-1} \partial_{r}-r^{-2} \partial_{\varphi}^{2}\right) t_{\mu}^{\lambda}=-\lambda^{2} t_{\mu}^{\lambda} . \\
K t_{\mu}^{\lambda}=\left(\xi_{1}^{2}+\xi_{2}^{2}\right) t_{\mu}^{\lambda}=\left(\partial_{r}^{2}+r^{-1} \partial_{r}+r^{-2} \partial_{\varphi}^{2}\right) t_{\mu}^{\lambda}=-\lambda^{2} t_{\mu}^{\lambda} .
\end{gathered}
$$

From (27), (28), (29) it follows $t_{\mu}^{\lambda}=(-c)^{\mu} e^{c \mu \varphi} f^{\lambda}(r)$ where $f^{\lambda}(r)$ satisfies the corresponding equations

$$
\left(\partial_{r}^{2}+r^{-1} \partial_{r}-\lambda^{2}-\mu^{2} r^{-2}\right) f^{\lambda}=0, \quad\left(\partial_{r}^{2}+r^{-1} \partial_{r}+\lambda^{2}-\mu^{2} r^{-2}\right) f^{\lambda}=0 .
$$

The solution of the first equation in (30) is the McDonald function $f^{\lambda}=K_{\mu}(\lambda r)$, and of the second is the cylindrical function $f^{\lambda}=Z_{\mu}(\lambda r)$ [10]. Thus the scalar harmonics can be expressed in the form

$$
\begin{aligned}
t_{\mu}^{\lambda} & =(-i)^{\mu} e^{\mu \varphi}\left(C_{1} K_{\mu}(\lambda r)+C_{2} K_{-\mu}(\lambda r)\right), \\
t_{\mu}^{\lambda} & =(-i)^{\mu} e^{i \mu \varphi}\left(C_{1} Z_{\mu}(\lambda r)+C_{2} Z_{-\mu}(\lambda r)\right) .
\end{aligned}
$$

It is important for further application that the particular solutions of the second equation in (30) are the Hankel functions of the first and second orders. The tensor symmetric harmonic of weight $\lambda$ and type $(0,2)$ are expressed in the form

$$
T^{\lambda \mu}=T_{i k}^{(\lambda \mu)} e^{i} \otimes e^{k}+T_{j s}^{(\lambda \mu)}\left(e^{j} \otimes e^{s}+e^{s} \otimes e^{j}\right)+T_{s s^{\prime}}^{(\lambda \mu)} e^{s} \otimes e^{s^{\prime}},
$$

where the basic vectors $e^{i}$ are invariant under the group transformations, and the basis $e^{s},\left(s, s^{\prime}=1,2\right)$ on the surface of transitivity dual to that defined by (26) is

$$
e^{1}=\frac{1}{2} e^{-c \varphi}\left(d r+\frac{r}{c} d \varphi\right), \quad e^{2}=\frac{1}{2} e^{c \varphi}\left(d r-\frac{r}{c} d \varphi\right) .
$$

The irreducible components can be written in the form

$$
T_{i k}^{(\lambda \mu)}=h_{i k} t^{(\lambda \mu)} ; \quad T_{j s}^{(\lambda \mu)}=h_{j s} t^{(\lambda \mu)} ; \quad T_{s s^{\prime}}^{(\lambda \mu)}=h_{s s^{\prime}}^{(\lambda \mu)} t^{(\lambda \mu)} .
$$

From the above and the well-known recurrent relations for scalars [10] we obtain the corresponding relations for tensors.

(b) $G_{3} V I I I$ and $G_{3} I X$ on $M^{2}$. Here we have the following sets of the Killing vectors:

$$
\begin{aligned}
& \xi_{1}=\cosh y \partial_{x}-\sinh y \operatorname{coth} x \partial_{y}, \quad \xi_{2}=\sinh y \partial_{x}-\cosh y \operatorname{coth} x \partial_{y}, \quad \xi_{3}=\partial_{y} \\
& \xi_{1}=-\cos y \partial_{x}+\sin y \operatorname{coth} x \partial_{y}, \quad \xi_{2}=\sin y \partial_{x}+\cos y \operatorname{coth} x \partial_{y}, \quad \xi_{3}=-\partial_{y}, \\
& \xi_{1}=-\cos y \partial_{x}+\sin y \cot x \partial_{y}, \quad \xi_{2}=\sin y \partial_{x}+\cos y \cot x \partial_{y}, \quad \xi_{3}=-\partial_{y}, \\
& \xi_{1}=\cosh y \partial_{x}-\sinh y \cot x \partial_{y}, \quad \xi_{2}=\sinh y \partial_{x}-\cosh y \cot x \partial_{y}, \quad \xi_{3}=-\partial_{y} .
\end{aligned}
$$

The groups generated by these operators are semi-simple and non-integrable. The Cartan tensor is non-degenerate in all these case, and $\gamma^{i k}=\operatorname{diag}(-1,1,1), \gamma^{i k}=\operatorname{diag}(1,1,-1), \gamma^{i k}=\operatorname{diag}(1,1,1), \gamma^{i k}=\operatorname{diag}(-1,1,1)$.

Go over from $\xi_{1}, \xi_{2}$ to the creation and annihilation operators

$$
H_{s}=e^{s y}\left(s \partial_{x}+\alpha \operatorname{coth} x \partial_{y}\right)
$$

where $s= \pm 1, \alpha=-1$ and $s= \pm i, \alpha=-1$ for the groups generated by the operators (35) and (36) respectively, and

$$
H_{s}=e^{s y}\left(s \partial_{x}+\alpha \cot x \partial_{y}\right),
$$


where $s= \pm \imath, \alpha=-1$ and $s= \pm 1, \alpha=-1$ for the groups generated by the operators (37) and (38) respectively.

The operators $L_{H_{S}}$ are the creation and annihilation operators for tensor functions $\hat{T_{B}^{A}}$, which, in their turn, are the eigenfunctions of the operator $L_{H_{3}}$. In the spirit of the book [10] we can show that there is the set of tensor functions $T_{(m) B}^{(l) A}$ for which

$$
\begin{gathered}
\mathcal{L}_{H_{3}} T_{(m) B}^{(l) A}=m T_{(m) B}^{(l) A} \quad(m=-l,-l+1, \ldots, l) ; \\
\mathcal{L}_{H_{S}} T_{(m) B}^{(l) A}=\sqrt{l(l+1)-m(m+s)} T_{(m) B}^{(l) A},
\end{gathered}
$$

where $l$ is the weight of the representation. Herewith the tensor eigenfunction equations (5) can be written in the form

$$
-\mathcal{G} T_{(m) B}^{(l) A}=l(l+1) T_{(m) B}^{(l) A}
$$

where $\mathcal{G}$ is the generalized Casimir operator.

Suppose that we need to consider the tensor symmetric harmonics of type $(2,0)$ and of weight $l$, which we shall denote $T^{l}$. With respect to the initial differential basis $e^{z}, d x^{1}=d x, d x^{2}=d y$ they can be written in the form

$$
T^{l}=T_{z z}^{(l)} e^{z} \otimes e^{z}+T_{r a}^{(l)}\left(e^{z} \otimes d x^{a}+d x^{a} \otimes e^{z}\right)+T_{a b}^{(l)} d x^{a} \otimes d x^{b} .
$$

Note, that the covector $e^{z}=d z$ is invariant with respect to these groups. In order to split the system of equations (43) for the tensor (44), into the irreducible components, one should go over into the basis of one-forms on the surfaces of transitivity $M^{2}$ satisfying the condition (12).

It turns out that the covectors

$$
e^{s}=d x+s \sin x d y ; \quad e^{s}=d x+s \sinh x d y ; \quad(s \in \pm 1, \imath)
$$

are required for the groups generated by the operators (35), (36) and (37), (38) respectively. The condition of invariance of a split structure (9) for the Lie operators associated with the vectors (39) stipulates the corresponding relations

$$
\mu_{s^{\prime}}^{s}=\frac{s s^{\prime} e^{i s^{\prime} y}}{\sin x} ; \quad \mu_{s^{\prime}}^{s}=\frac{s s^{\prime} e^{i s^{\prime} y}}{\sinh x} ; \quad \mu_{3}^{s}=0 \quad\left(s, s^{\prime}= \pm 1\right)
$$

By using (45) the relation (44) can be rewritten in the form

$$
T^{l}=T_{z z}^{(l)} e^{z} \otimes e^{z}+T_{z s}^{(l)}\left(e^{r} \otimes e^{s}+e^{s} \otimes e^{z}\right)+T_{s s^{\prime}}^{(l)} e^{s} \otimes e^{s^{\prime}} .
$$

where the sum on $s= \pm 1$ is implied. Then, if we suppose

$$
T_{z z}^{(l)}=h_{z z} t^{l} ; \quad T_{z s}^{(l)}=h_{z} t_{s}^{l} ; \quad T_{s^{\prime} s}^{(l)}=h t_{s+s^{\prime}}^{l}
$$

where $h_{z z}, h_{z}, h$ are functions of $z$, then for the function $t_{n}^{l}\left(n=0, \ldots, s, \ldots, s+s^{\prime}\right)$ we obtain

$$
\left\{\frac{1}{\sinh x} \frac{\partial}{\partial x} \sinh x \frac{\partial}{\partial x}+\frac{1}{\sinh ^{2} x}\left(\frac{\partial^{2}}{\partial y^{2}}-2 i n \cosh x \frac{\partial}{\partial y}-n^{2}\right)+l(l+1)\right\} t_{n}^{l}=0
$$

for groups generated by the Killing vectors (36), and exactly the same equation with ordinary $\cos x, \sin x$ instead of the hyperbolic ones for (37). For the other two cases we obtain

$$
\left\{\frac{1}{\sinh x} \frac{\partial}{\partial x} \sinh x \frac{\partial}{\partial x}-\frac{1}{\sinh ^{2} x}\left(\frac{\partial^{2}}{\partial y^{2}}-2 n \cosh x \frac{\partial}{\partial y}+n^{2}\right)+l(l+1)\right\} t_{n}^{l}=0 .
$$

for (35) and exactly the same equation with the corresponding ordinary trigonometric functions for (38).

Owing to (41) we find the general solutions for groups generated by the Killing vectors (35), (36), and (37), (38) respectively

and

$$
t_{n}^{l}=\Sigma_{m} C_{m} e^{c m y} \mathfrak{B}_{n m}^{l}
$$

$$
t_{n}^{l}=\Sigma_{m} C_{m} e^{c m y} P_{n m}^{l}
$$

where $c=1$ for (35), (38) and $c=i$ for (36), (37), $C_{m}$ are some coefficients. Here the function $\mathfrak{B}_{n m}^{l}$ satisfies the corresponding differential equation following from (49):

$$
\left\{\frac{1}{\sinh x} \frac{d}{d x} \sinh x \frac{d}{d x}-\frac{m^{2}-2 m n \cosh x+n^{2}}{\sinh ^{2} x}+l(l+1)\right\} \mathfrak{B}_{n m}^{l}=0,
$$

and $P_{n m}^{l}$ satisfies exactly the same equation with ordinary $\cos x, \sin x$. The solutions of the obtained equations are the functions $\mathfrak{B}_{n m}^{l}(\cosh x)$ and $P_{n m}^{l}(\cos x)$ and, which are called the Legendre functions and Legendre polynomials respectively [10]. Recurrent relations for them follow from (41), (42).

It is obvious that in a similar fashion we can treat all the other types of gravity in Petrov classification scheme. 


\section{Applications}

\subsection{Weak cylindrical gravitational waves as perturbations of the flat space-time}

Now as a quick illustration of the previous section we shall consider the background metric of a flat space-time in the form

$$
d s^{2}=d t^{2}-d r^{2}-r^{2} d \varphi^{2}-d z^{2} .
$$

Except $\partial_{t} \partial_{z}$ it has the Killing's vectors (24) and thus can be expanded with the help of the cylindrical harmonics (31). The representation basis of the full space-time according to (26) is

$$
e_{0}=\partial_{t}, \quad e_{1}=e^{i \varphi}\left(\partial_{r}+\frac{i}{r} \partial_{\varphi}\right), \quad e_{2}=e^{-i \varphi}\left(\partial_{r}-\frac{i}{r} \partial_{\varphi}\right), \quad e_{3}=\partial_{z}
$$

Then according to (62) we almost immediately obtain the irreducible combinations of the linearized Einstein equations (63 - 69) writing the perturbations of the Ricci tensor with respect to the representation basis. Since these equations are invariant under the change of a sign of time, i.e. since the parity operator commutes with all the other operators of the underlying group one can write the perturbations $h_{\mu \nu}$ as the sum of parts associated with positive and negative frequencies $\pm \omega= \pm \sqrt{k^{2}+\lambda^{2}}$ :

$$
h_{a b}(x)=h_{a b}^{+}(x)+h_{a b}^{-}(x),
$$

where $h_{a b}^{ \pm}(x)$ are expanded into the series

$$
h_{a b}^{ \pm}(x)=\int_{-\infty}^{+\infty} \exp ( \pm i \omega t \mp i k z) d k \sum_{n=-\infty}^{n=+\infty} \int_{0}^{+\infty} a_{a b}^{ \pm}(x) \Phi_{n}^{ \pm} \lambda d \lambda .
$$

Here $\Phi_{n}^{ \pm}$being an eigenfunction of the Casimir operator with the eigenvalue $-\lambda^{2}$ satisfies the relations

$$
K \Phi_{n}^{ \pm}=-\lambda^{2} \Phi_{n}^{ \pm}, \quad e_{1} \Phi_{n}^{ \pm}= \pm i \lambda \Phi_{n \pm 1}^{ \pm}, \quad e_{2} \Phi_{n}^{ \pm}= \pm i \lambda \Phi_{n \mp 1}^{ \pm}, \quad \Phi_{n}^{+}=\left(\Phi_{n}^{-}\right)^{*}
$$

where $e_{1}, e_{2}$ are determined by (26), and $K$ by (29).

Then by analogy with electrodynamics if we choose the Bessel function as the solution of (30) we easily find the system of algebraic equations (see Appendix [70]- 730) describing the standing waves in the $r-\varphi$ plane [11, and if the Hankel function - the running cylindrical waves. These equations are equivalent to the coordinate conditions

$$
\Gamma_{\alpha \beta \gamma} g^{\alpha \beta}=\frac{1}{r} h_{1 \gamma},
$$

where all the objects are determined with respect to the cylindrical coordinates basis $d t, d r, d \varphi, d z$ (remember that $\alpha, \beta, \mu, \nu, \ldots$ are the coordinates indices). Then we can use the gauge freedom to decrease the number of independent coefficients $\left(a_{a b}\right)_{n}$.

\subsection{Spherical symmetry: Regge - Wheller harmonics}

Consider the background metric in the Schwarzshild form

$$
d s^{2}=-\left(1-\frac{2 m^{*}}{r}\right) d t^{2}+\left(1-\frac{2 m^{*}}{r}\right)^{-1} d r^{2}+r^{2}\left(d \theta^{2}+\sin ^{2} \theta d \varphi^{2}\right)
$$

The Schwarzshild space-time being stationary and spherically symmetric admits the four Killing vectors: the three defined in (37) and $\partial_{t}$. Hence it follows that the basis

$$
e_{0}=\partial_{t}, \quad e_{1}=\partial_{r}, \quad e_{2}=\frac{1}{2}\left(\partial_{\theta}-\frac{i}{\sin \theta} \partial_{\varphi}\right), \quad e_{3}=\frac{1}{2}\left(\partial_{\theta}+\frac{i}{\sin \theta} \partial_{\varphi}\right)
$$

can be chosen as a representation basis. The Christoffel symbols evaluated at the pole with respect to this basis are given by the formulas

Substituting The Christoffel symbols evaluated at the pole with respect to this basis for those in (62) and using expansion into spherical harmonics $P_{m n}^{l}$ at $m=0$ after the Regge-Wheeler gauge transformations in the representation basis (61) we can write the perturbations of the Ricci tensor with respect to this basis, i.e.in the form of the irreducible combinations of the linearized Einstein equations $\delta R_{a b}=0$, and thus separate radial variable and time from the angular variables. The eventual equations are equivalent to those of the works [2] and [12] and therefore are not listed here. The basis (61), that can be multiplied by an arbitrary function of $r$ and $t$, are widely used (see for example [13]) and the tensor harmonics following from it were discussed by many authors [14, 15] . 


\section{Acknowledgement}

I would like to acknowledge Professor V.D.Gladush for proposing the problem, much encouragement and reading the manuscript. I am also much indebted to Dr. S.Ulanov for computer help.

\section{Appendix}

\section{A The linearized Einstein equations for empty space with respect to arbitrary basis}

The relation (22) written out in terms of the Christoffel symbols and of the basis vectors reads

$$
\begin{aligned}
2 \delta R_{a b}= & g^{a b}\left[e_{a} e_{b} h_{m n}-e_{n} e_{b} h_{m a}-e_{m} e_{b} h_{n a}+e_{b}\left(\left(\Gamma_{m n}^{l}+\Gamma_{n m}^{l}\right) h_{l a}\right.\right. \\
& \left.+\left(\Gamma_{a n}^{l}-\Gamma_{n a}^{l}\right) h_{l m}+\left(\Gamma_{a m}^{l}-\Gamma_{m a}^{l}\right) h_{l n}\right)+\Gamma_{a b}^{l}\left(e_{n} h_{m l}+e_{m} h_{n l}-e_{l} h_{m n}\right) \\
& +\Gamma_{m b}^{l}\left(e_{n} h_{l a}+e_{l} h_{n a}-e_{a} h_{l n}\right)-\left(\Gamma_{m b}^{k}\left(\Gamma_{k n}^{l}+\Gamma_{n k}^{l}\right)+\Gamma_{n b}^{k}\left(\Gamma_{m k}^{l}+\Gamma_{k m}^{l}\right)\right) h_{l a}+ \\
& +\Gamma_{n b}^{k}\left(e_{m} h_{k a}+e_{k} h_{m a}-e_{a} h_{k m}\right)+\left(\Gamma_{m b}^{k}\left(\Gamma_{k a}^{l}-\Gamma_{a k}^{l}\right)+\Gamma_{a b}^{k}\left(\Gamma_{k m}^{l}-\Gamma_{m k}^{l}\right)\right) h_{l n} \\
& \left.-\Gamma_{a b}^{k}\left(\Gamma_{m n}^{l}+\Gamma_{n m}^{l}\right)+\left(\Gamma_{a b}^{k}\left(\Gamma_{n k}^{l}-\Gamma_{k n}^{l}\right)+\Gamma_{n b}^{k}\left(\Gamma_{k a}^{l}-\Gamma_{a k}^{l}\right)\right) h_{m l}\right]+e_{m} e_{n} h-\Gamma_{m n}^{l} e_{l} h=0,
\end{aligned}
$$

where $a, b, m, n, k, l, \ldots=0,1,2,3$ are indices associated with the basis vectors $e_{0}, e_{1}, \ldots$, so that $g_{a b}, \Gamma_{b c}^{a}$, and $h_{a b}=h_{b a}$ are the background metric, the Christoffel symbols, and the perturbations determined with respect to the basis $e_{a}(a=i, s=0,1,2,3)$.

\section{B The linearized Einstein equations for cylindrical waves as pertur- bations of the flat space-time in the form of the irreducible combi- nations}

$$
\begin{aligned}
\delta R_{00}= & -\frac{1}{2}\left(e_{1} e_{2}+e_{3} e_{3}\right) h_{00}+\frac{1}{2} e_{0} e_{2} h_{01}+e_{0} e_{1} h_{02}+e_{0} e_{3} h_{03}-e_{0} e_{0}\left(h_{12}+h_{33}\right), \\
\delta R_{01}= & -\frac{1}{2}\left(-\frac{1}{2} e_{1} e_{2}-e_{3} e_{3}\right) h_{01}+\frac{1}{4} e_{1} e_{1} h_{02}+\frac{1}{2} e_{1} e_{3} h_{03}+\frac{1}{4} e_{0} e_{2} h_{11}-\frac{1}{4} e_{0} e_{1} h_{12} \\
& +\frac{1}{2} e_{0} e_{3} h_{13}-\frac{1}{2} e_{0} e_{1} h_{33}=0 \\
\delta R_{03}= & \frac{1}{4} e_{3} e_{2} h_{01}+\frac{1}{4} e_{3} e_{1} h_{02}-\frac{1}{2} e_{1} e_{2} h_{03}-\frac{1}{2} e_{0} e_{3} h_{12}+\frac{1}{4} e_{0} e_{2} h_{13}+\frac{1}{4} e_{0} e_{1} h_{23}=0 \\
\delta R_{13}= & \frac{1}{2} e_{1} e_{3} h_{00}-\frac{1}{2} e_{0} e_{3} h_{01}-\frac{1}{2} e_{0} e_{1} h_{03}+\frac{1}{4} e_{3} e_{2} h_{11}-\frac{1}{4} e_{1} e_{3} h_{12}+\frac{1}{2}\left(e_{0} e_{0}-\frac{1}{2} e_{1} e_{2}\right) h_{13} \\
& +\frac{1}{4} e_{1} e_{1} h_{23}=0 \\
\delta R_{12}= & \frac{1}{2} e_{1} e_{2} h_{00}-\frac{1}{2} e_{0} e_{2} h_{01}-\frac{1}{2} e_{0} e_{1} h_{02}+\frac{1}{4} e_{2} e_{2} h_{11}+\frac{1}{2}\left(e_{0} e_{0}-e_{1} e_{2}-e_{3} e_{3}\right) h_{12}+\frac{1}{2} e_{2} e_{3} h_{13} \\
& +\frac{1}{4} e_{1} e_{1} h_{22}+\frac{1}{2} e_{1} e_{3} h_{23}-\frac{1}{2} e_{1} e_{2} h_{33}=0, \\
\delta R_{11}= & \frac{1}{2} e_{1} e_{1} h_{00}-e_{1} e_{0} h_{01}+\frac{1}{2}\left(e_{0} e_{0}-e_{3} e_{3}\right) h_{11}+e_{1} e_{3} h_{13}-\frac{1}{2} e_{1} e_{1} h_{33}=0, \\
\delta R_{33}= & \frac{1}{2} e_{3} e_{3} h_{00}-e_{0} e_{3} h_{03}-\frac{1}{2} e_{3} e_{3} h_{12}+\frac{1}{2} e_{3} e_{2} h_{13}+\frac{1}{2} e_{3} e_{1} h_{23}+\frac{1}{2}\left(e_{0} e_{0}-e_{1} e_{2}\right) h_{33}=0 .
\end{aligned}
$$

\section{The algebraic equations describing the cylindrical waves}

$$
\begin{gathered}
\omega\left(a_{00}\right)_{n}+\lambda\left[\left(a_{01}\right)_{n+1}+\left(a_{02}\right)_{n-1}\right]+2 k\left(a_{03}\right)_{n}+\omega\left(a_{12}\right)_{n}+\omega\left(a_{33}\right)_{n}=0, \\
\lambda\left[\left(a_{33}\right)_{n}-\left(a_{00}\right)_{n}-\left(a_{11}\right)_{n+2}\right]-2 \omega\left(a_{01}\right)_{n+1}-2 k\left(a_{13}\right)_{n+1}=0, \\
\lambda\left[\left(a_{33}\right)_{n}-\left(a_{00}\right)_{n}-\left(a_{22}\right)_{n-2}\right]-2 \omega\left(a_{02}\right)_{n-1}-2 k\left(a_{23}\right)_{n-1}=0, \\
\lambda\left[\left(a_{00}\right)_{n}-\left(a_{12}\right)_{n}+\left(a_{33}\right)_{n}\right]+2 \omega\left(a_{03}\right)_{n}+\lambda\left[\left(a_{13}\right)_{n+1}+\left(a_{23}\right)_{n-1}\right]=0, \\
a_{a b}^{+}(k, n, \lambda) \equiv\left(a_{a b}\right)_{n} .
\end{gathered}
$$




\section{References}

[1] E.P.Wigner, Group theory (New York, 1959).

[2] T.Regge and J.A.Wheller, Phys.Rev. 108, 1063 (1957).

[3] E.M.Lishitz, J.Phys. (USSR) 10, 116 (1946).

[4] R.Gleiser, O.Nicasio, R.H.Price and J.Pullin to appear in Phys.Rep. (1999) (gr-qc 9807077).

[5] V.D.Gladush and S.Yu.Gorbachev, Ukr.Fiz.J. N11-12, Vol.40, 1244 (1995) (in Ukrainian).

[6] V.D.Gladush and R.A.Konoplya, J.Math. Phys. 40, 955, (1999).

[7] S.Weinberg, Gravitation and Cosmology (ed. John Wiley and Sons, New York, 1972).

[8] A.Z.Petrov, New Methods in General Relativity (Moskow, Nauka, 1966) (in Russian).

[9] A.Z.Petrov, Einstein spaces (Pergamon Press, 1969).

[10] N.Ya.Vilenkin. Special Functions and The Theory of Group Representations. Moskow, Nauka, (1991) (in Russian).

[11] V.E.Radchenko, Izv.Vyssh.Uchebn.Zaved.Fiz N5, 7 (1975) (in Russian).

[12] F.J.Zerilly, Phys.Rev.Lett. 24, 737 (1970).

[13] E.G.Kalnins and W.Miller,Jr., J.Math. Phys. 32, 698, (1991).

[14] F.J.Zerilly, J.Math. Phys. 11, 2203, (1970).

[15] B.V.Hu, J.Math. Phys. 15, 1748, (1974). 